JULY, 1974

\title{
SKEW CRACK PROPAGATION AT THE DIFFRACTION OF A TRANSIENT STRESS WAVE*
}

\author{
$\mathrm{BY}$ \\ J. D. ACHENBACH AND V. K. VARATHARAJULU \\ Northwestern University
}

1. Introduction. When a stress wave strikes a crack, a stress field is generated which contains square-root singularities at the tips of the crack. The question of fracture under the influence of such a stress field was investigated in [1] and [2] for anti-plane and in-plane motions, respectively. In both [1] and [2] a semi-infinite crack in a twodimensional geometry was considered, and it was assumed that the crack propagates in its own plane. The principal task of the analysis of [1] and [2] consisted in solving an unusual transient diffraction problem, namely, the diffraction of an elastic wave by a semi-infinite crack which starts to extend after the crack tip has been struck.

In this paper we also consider transient diffraction of an elastic wave by an extending crack, but with the distinction that the crack may propagate under an arbitrary angle with its own plane. The incident wave is a plane horizontally-polarized wave. It is assumed that crack propagation at a constant velocity is generated at the instant that the tip of a semi-infinite crack is struck. An expression is derived for the stress intensity factor in terms of the speed of crack propagation and the angle of crack propagation.

For a step-stress incident wave a solution for the particle velocity is sought which shows dynamic similarity inside the circular region of the diffracted wave. A crucial step in the analysis is the use of Chaplygin's transformation, which reduces the problem to the solution of Laplace's equation in a semi-infinite strip containing a slit. Other applications in elastodynamics of dynamic similarity and Chaplygin's transformation can be found in [3] and [4]. The Schwarz-Christoffel transformation is subsequently employed to map the semi-infinite strip on a half-plane. The appropriate analytic function in the half-plane is obtained by a method which was discussed in great detail by Muskhelishvili [5].

Expressions for the shear stresses and the particle velocity in the vicinity of the crack tip were obtained by means of a limiting process which is analogous to the one worked out by Sih [6] for problems of static equilibrium. The interesting aspect of the results is that the dependence of the near-tip fields on the angle of crack propagation separates from the dependence on a polar angle centered at the moving crack tip.

2. The diffraction problem. Horizontally polarized wave motion in a homogeneous, isotropic, linearly elastic solid is governed by the two-dimensional wave equation

\footnotetext{
* Received January 20, 1973; revised version received February 20, 1973. This work was supported by the Advanced Research Projects Agency of the U.S. Department of Defense through the Northwestern University Materials Research Center.
} 


$$
\partial^{2} w / \partial x^{2}+\partial^{2} w / \partial y^{2}=\left(1 / c_{T}{ }^{2}\right) \partial^{2} w / \partial t^{2}
$$

where $w(x, y, t)$ is the displacement normal to the $x-y$ plane and $c_{T}$ is the velocity of transverse waves,

$$
c_{T}=(\mu / \rho)^{1 / 2},
$$

where $\mu$ and $\rho$ are the shear modulus and the mass density, respectively.

At time $t=0$ a plane incident wave of the form

$$
w_{\mathrm{inc}}(x, y, t)=f(\tau)
$$

where

$$
\tau=t+\left(x / c_{T}\right) \sin \alpha-\left(y / c_{T}\right) \cos \alpha
$$

and $f(\tau) \equiv 0$ for $\tau \leq 0$, strikes the tip of a semi-infinite crack. The position of the wavefront prior to time $t=0$ is shown in Fig. 1a. It is assumed that the crack starts to propagate at a constant velocity $v$, where $v / c_{T}<1$, at the instant that the crack tip is struck. In contradistinction to earlier work, the crack is, however, not assumed to propagate in its own plane, but rather in a plane which makes an angle $\kappa \pi$ with the plane of the crack (see Fig. 1b). Thus, at time $t>0$ the crack tip is located at point $D$ which is
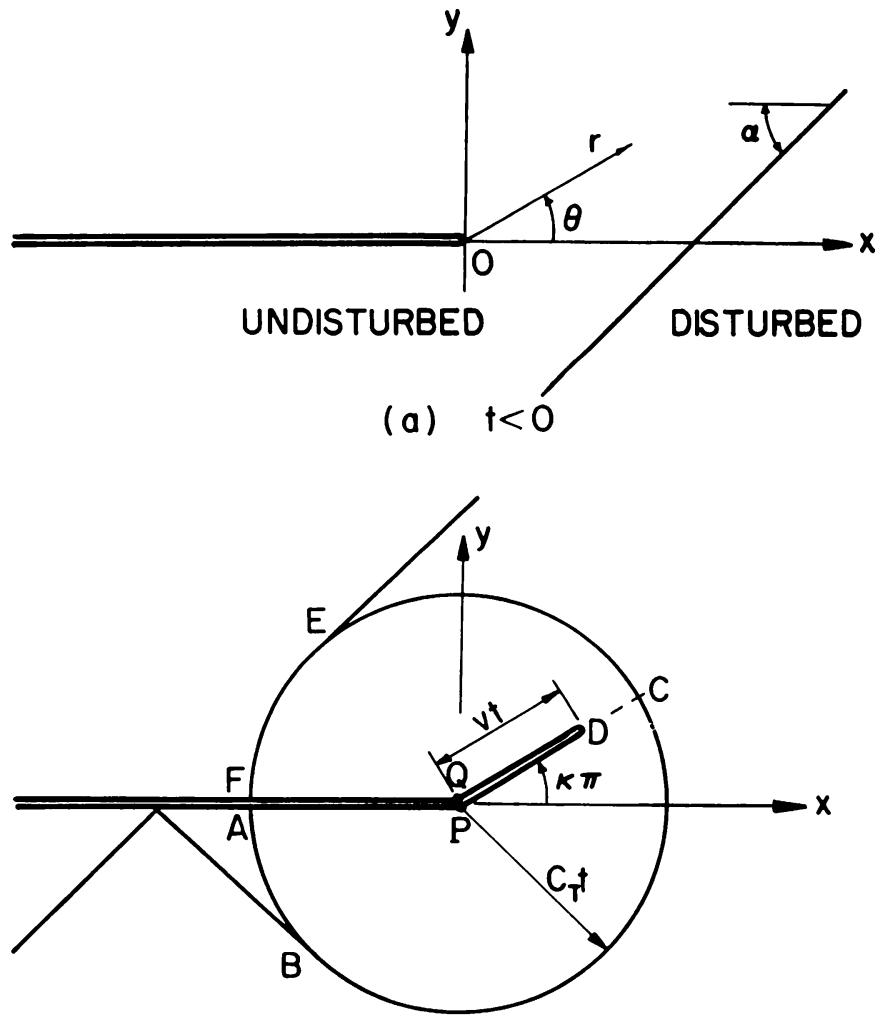

(b) $\quad t>0$

FIG. 1. Pattern of wavefronts and position of the crack tip. 
defined by $r=v t, \theta=\kappa \pi$. At time $t$ the crack has generated a plane reflected wave and a cylindrical diffracted wave. The pattern of wavefronts and the position of the crack tip is shown in Fig. 1b. Note that $P$ and $Q$ denote points which coincide with the origin 0 but which are located on the lower and upper surfaces of the crack, respectively.

The reflection and diffraction of the incident wave involve horizontally polarized motions only which are governed by Eq. (2.1). To investigate the cylindrical wave it is convenient to express the Laplacian in polar coordinates, with fixed origin at $\mathbf{0}$. Eq. (2.1) is thus rewritten as

$$
\frac{1}{r} \frac{\partial}{\partial r}\left(r \frac{\partial w}{\partial r}\right)+\frac{1}{r^{2}} \frac{\partial^{2} w}{\partial \theta^{2}}=\frac{1}{c_{T}^{2}} \frac{\partial^{2} w}{\partial t^{2}} .
$$

The relevant shear stresses are

$$
\tau_{r z}=\mu \partial w / \partial r, \quad \tau_{\theta z}=(\mu / r) \partial w / \partial \theta .
$$

In this paper we will consider the case of a step-stress incident wave. The function $f(\tau)$ in Eq. (2.3) then is of the form

$$
f(\tau)=W_{0} \tau H(\tau)
$$

where $H(\tau)$ is the Heaviside step function. The method of solution of Eq. (2.5) is based on the premise that certain field variables should show dynamic similarity, since there is no characteristic length in this diffraction problem. This implies that these field variables depend on the independent variables $r, \theta$ and $t$ only through dependence on $\theta$ and on the ratio $r / t$. For an incident wave of the form (2.7) the particle velocity

$$
W=\partial w / \partial t
$$

shows this property, i.e., $W(r, \theta, t)=W(s, \theta)$, where

$$
s=r / t \text {. }
$$

To solve for $W(s, \theta)$ it is convenient to introduce $s=r / t$ as a new variable. The equation governing $W(s, \theta)$ follows from Eq. (2.1) as

$$
s^{2}\left(1-\frac{s^{2}}{c_{T}^{2}}\right) \frac{\partial^{2} W}{\partial s^{2}}+s\left(1-\frac{2 s^{2}}{c_{T}^{2}}\right) \frac{\partial W}{\partial s}+\frac{\partial^{2} W}{\partial \theta^{2}}=0 .
$$

In terms of $s$ and $\theta$ the boundary conditions on $W(s, \theta)$ take the form (see Fig. 1a, b):

$$
\begin{aligned}
\theta=-\pi, & s \leq c_{T}, & \partial W / \partial \theta & =0, \\
-\pi \leq \theta<-(\alpha+\pi / 2), & s=c_{T}, & W & =2 W_{0}, \\
-(\alpha+\pi / 2)<\theta<\alpha+\pi / 2, & s=c_{T}, & W & =W_{0}, \\
\alpha+\pi / 2<\theta \leq \pi, & s=c_{T}, & W & =0, \\
\theta=\pi, & s \leq c_{T}, & \partial W / \partial \theta & =0, \\
\theta=\kappa \pi \pm \epsilon, & s<v, & \partial W / \partial \theta & =0 .
\end{aligned}
$$

For $s \leq c_{T}$, Eq. (2.10) is elliptic. By means of Chaplygin's transformation

$$
\beta=\operatorname{arcosh}\left(c_{T} / s\right),
$$


Eq. (2.10) can then be simplified to Laplace's equation

$$
\partial^{2} W / \partial \beta^{2}+\partial^{2} W / \partial \theta^{2}=0 .
$$

The real transformation (2.17) maps the circular domain $s \leq c_{T},-\pi \leq \theta \leq \pi$, into a semi-infinite strip containing a slit in the $\theta-\beta$ plane (see Fig. 2). Inside this domain $W(\beta, \theta)$ satisfies Eq. (2.18). The boundary conditions are indicated in Fig. 2.

Within the semi-infinite strip in the $\theta-\beta$ plane the solution of Laplace's equation may be written as the real part of an analytic function $G(\gamma)$

$$
W=\operatorname{Re} G(\gamma)
$$

where

$$
\gamma=\beta+i \theta
$$

Eq. (2.19) implies

$$
T_{p z}-i T_{\psi z}=\mu G^{\prime}(\gamma) \exp (i \psi)
$$

where

$$
T_{p z}=\partial \tau_{p z} / \partial t, \quad T_{\psi z}=\partial \tau_{\psi z} / \partial t .
$$

Here $p$ and $\psi$ are polar coordinates centered at the point $D$ in the $\theta-\beta$ plane (see Fig. 2) and $\tau_{p z}$ and $\tau_{\psi z}$ are defined analogously to Eqs. $(2.6 \mathrm{a}, \mathrm{b})$. A prime denotes a derivative with respect to the argument of the function.

The function $G(\gamma)$ can, in principle, be obtained by the use of conformal mapping techniques. The region in the $\gamma$-plane can be mapped on the upper half of the $\zeta$-plane, where $\zeta=\xi+i \eta$, by the following Schwarz-Christoffel transformation:

$$
\gamma=\omega(\zeta)=C_{1} \int_{1}^{\zeta} \frac{(u-\kappa) d u}{\left(u+\xi_{P}\right)\left(u-\xi_{Q}\right)\left(1-u^{2}\right)^{1 / 2}}+C_{2}
$$

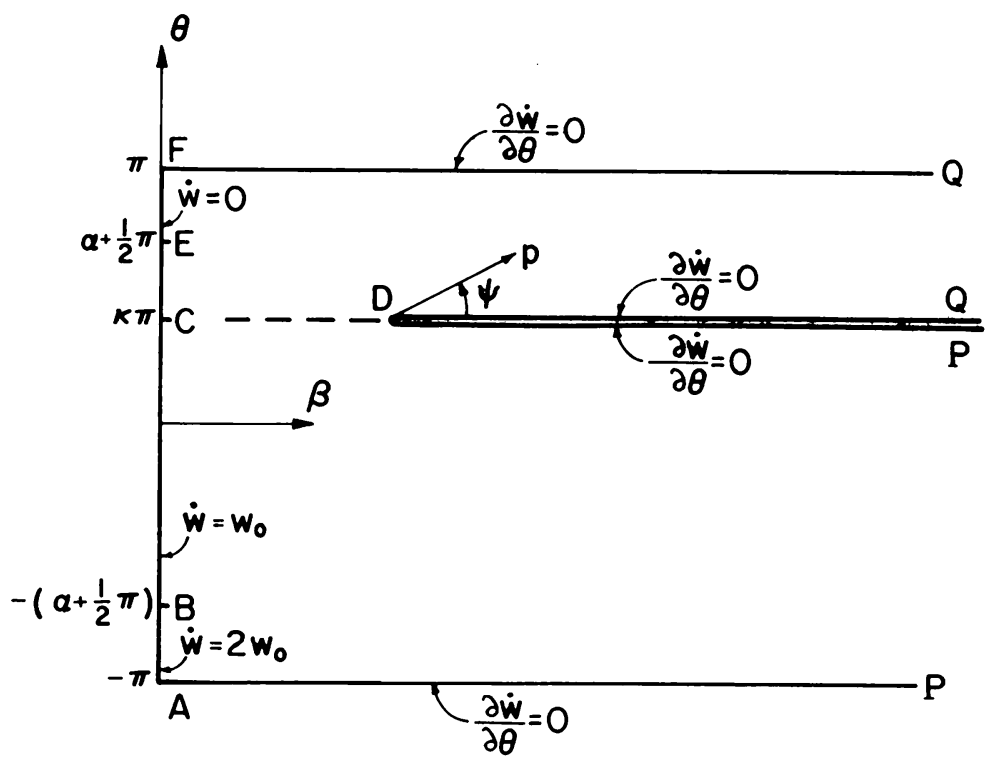

Fig. 2. Domain in the $\theta-\beta$ plane. 


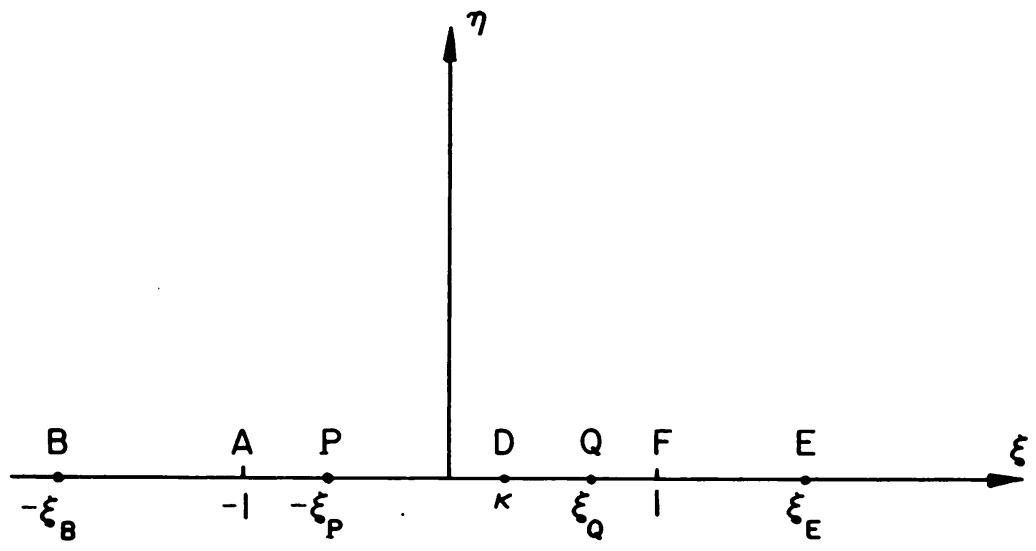

Fig. 3. Mapping on $\zeta$-plane.

where $C_{1}$ and $C_{2}$ are complex constants. Schwarz-Christoffel transformations are discussed in some detail in [7]. The three arbitrary choices in the mapping are that the points $A, F$ and $D$ are mapped into the points $\zeta=-1, \zeta=+1$ and $\zeta=\kappa$, respectively. The $\zeta$-plane is shown in Fig. 3.

The integral in Eq. (2.23) can be evaluated to yield

$$
\begin{aligned}
\gamma= & -\frac{\xi_{P}+\kappa}{\xi_{P}+\xi_{Q}} \frac{C_{1}}{\left(1-\xi_{P}{ }^{1 / 2}\right.}\left\{\ln \left[\left(1-\xi_{P}{ }^{2}\right)^{1 / 2}\left(1-\zeta^{2}\right)^{1 / 2}+\zeta \xi_{P}+1\right]-\ln \left[\zeta+\xi_{P}\right]\right\} \\
& -\frac{\xi_{Q}-\kappa}{\xi_{P}+\xi_{Q}} \frac{C_{1}}{\left(1-\xi_{Q}{ }^{2}\right)^{1 / 2}}\left\{\ln \left[\left(1-\xi_{Q}{ }^{2 / 2}\left(1-\zeta^{2}\right)^{1 / 2}-\zeta \xi_{Q}+1\right]-\ln \left[\zeta-\xi_{Q}\right]\right\}\right. \\
& +C_{2} .
\end{aligned}
$$

The mapping of the point $F$ gives $C_{2}=i \pi$. By examining the changes of the imaginary parts at $P$ and $Q$ we obtain

$$
\begin{aligned}
& 1+\kappa=-\left(\xi_{P}+\kappa\right) C_{1} /\left(\xi_{P}+\xi_{Q}\right)\left(1-\xi_{P}^{2}\right)^{1 / 2}, \\
& 1-\kappa=-\left(\xi_{Q}-\kappa\right) C_{1} /\left(\xi_{P}+\xi_{Q}\right)\left(1-\xi_{Q}^{2}\right)^{1 / 2},
\end{aligned}
$$

respectively. Elimination of $C_{1}$ from (2.25) and (2.26) yields

$$
(1+\kappa) /(1-\kappa)=\left(\xi_{P}+\kappa\right)\left(1-\xi_{Q}{ }^{2}\right)^{1 / 2} /\left(\xi_{Q}-\kappa\right)\left(1-\xi_{P}{ }^{2}\right)^{1 / 2} .
$$

In view of Eqs. (2.25) and (2.26), the mapping can be rewritten as

$$
\begin{aligned}
\gamma= & (1+\kappa)\left\{\ln \left[\left(1-\xi_{P}{ }^{2}\right)^{1 / 2}\left(1-\zeta^{2}\right)^{1 / 2}+\zeta \xi_{P}+1\right]-\ln \left[\zeta+\xi_{P}\right]\right\} \\
& +(1-\kappa)\left\{\ln \left[\left(1-\xi_{Q}{ }^{2}\right)^{1 / 2}\left(1-\zeta^{2}\right)^{1 / 2}-\zeta \xi_{Q}+1\right]-\ln \left[\zeta-\xi_{Q}\right]\right\}+i \pi .
\end{aligned}
$$

Finally, comparing the coordinates of $D$ in the $\gamma$ and $\zeta$-planes, we obtain the following relation:

$$
\begin{aligned}
\ln \left\{c_{T} / v+\left[\left(c_{T} / v\right)^{2}-1\right]^{1 / 2}\right\} & \\
= & (1+\kappa) \ln \left\{\left[\left(1-\xi_{P}^{2}\right)^{1 / 2}\left(1-\kappa^{2}\right)^{1 / 2}+\kappa \xi_{P}+1\right] /\left(\xi_{P}+\kappa\right)\right\} \\
& +(1-\kappa) \ln \left\{\left[\left(1-\xi_{0}^{2}\right)^{1 / 2}\left(1-\kappa^{2}\right)^{1 / 2}-\kappa \xi_{Q}+1\right] /\left(\xi_{0}-\kappa\right)\right\} .
\end{aligned}
$$


Eqs. (2.27) and (2.29) allow us to compute $\xi_{P}$ and $\xi_{Q}$ for any given $\kappa$ and $v / c_{T}$. Values for $\xi_{B}$ and $\xi_{\Sigma}$ can be computed in the same manner from Eq. (2.28).

The boundary conditions shown in Fig. 2 are converted into conditions on the real axis in the $\zeta$-plane. If we write

$$
W=W_{0}+W_{s},
$$

the boundary conditions on $W_{s}$ at $\eta=0$ may be expressed as

$$
\begin{aligned}
& -\infty<\xi<-\xi_{B}, \quad \xi_{E}<\xi<\infty \quad W_{s}=0, \\
& -\xi_{B}<\xi<-1, \quad W_{\mathrm{s}}=W_{0}, \\
& -1 \leq \xi \leq 1 \text {, } \\
& 1<\xi<\xi_{E}, \\
& \partial W_{s} / \partial \eta=0 \text {, } \\
& W_{\mathrm{s}}=-W_{0} .
\end{aligned}
$$

The appropriate analytic function in the upper half of the $\zeta$-plane can be obtained by the method of sectionally holomorphic functions. This method has been discussed in great detail by Muskhelishvili [5]. For the present problem it is convenient to work with

$$
f(\zeta)=F^{\prime}(\zeta)=\partial W_{s} / \partial \xi-i \partial W_{s} / \partial \eta
$$

where

$$
W_{s}=\operatorname{Re} F(\zeta)
$$

and the Cauchy-Riemann conditions have been employed. The conditions on $W_{\mathrm{s}}$, Eqs. (2.31)-(2.34), now yield the following boundary conditions for $f(\zeta)$ :

$$
\begin{array}{lll}
\eta=0, & -1 \leq \xi \leq 1, & \operatorname{Im} f(\zeta)=0, \\
\eta=0, & |\xi|>1, & \operatorname{Re} f(\zeta)=0 .
\end{array}
$$

A problem with very similar conditions on the real axis was solved by Sih [8].

Since $\operatorname{Re} F(\zeta)$ is discontinuous at $\zeta=-\xi_{B}$ and $\zeta=\xi_{E}$, it is to be expected that $f(\zeta)$ will contain poles at these points. It is easily checked that the following expression for $f(\zeta)$ in the upper halfplane satisfies Eqs. (2.37) and (2.38):

$$
f(\zeta)=\frac{i}{\left(\zeta^{2}-1\right)^{1 / 2}}\left\{\frac{A}{\zeta+\xi_{B}}+\frac{B}{\zeta-\xi_{E}}\right\} .
$$

This expression has branch points at $\zeta= \pm 1$. The branch cuts are taken along the real axis, and we consider the branch of $\left(\zeta^{2}-1\right)^{1 / 2}$ which $\rightarrow \zeta$ as $|\zeta| \rightarrow \infty$. In view of Eqs. (2.35) and (2.36), $W_{\text {s }}$ is obtained as

$$
W_{s}=\operatorname{Re}\left\{\int_{-\infty}^{\zeta} \frac{i}{\left(u^{2}-1\right)^{1 / 2}}\left[\frac{A}{u+\xi_{B}}+\frac{B}{u-\xi_{E}}\right] d u+C\right\},
$$

where the path of integration is in the upper halfplane. To determine the constants $A, B$ and $C$, the integration in (2.40) is carried out along the real axis. The path of integration then consists of straight-line segments plus semicircular indentations in the upper halfplane when a pole is passed. The condition (2.31) along the real axis in the region $-\infty<\xi<-\xi_{B}$ shows that $C \equiv 0$. However, for $\xi>-\xi_{B}, W_{\text {s }}$ has a non-zero real part contributed by the integral along the semicircular portion of the path above $-\xi_{B}$. 
By applying the boundary condition (2.32) we find

$$
A=-\left(W_{0} / \pi\right)\left(\xi_{B}^{2}-1\right)^{1 / 2} .
$$

In the region $-1<\xi<1$, Eq. (2.40) yields

$$
W_{s}=W_{0}+\int_{-1}^{\zeta} \frac{1}{\left(1-u^{2}\right)^{1 / 2}}\left[\frac{A}{u+\xi_{B}}+\frac{B}{u-\xi_{E}}\right] d u .
$$

The condition $W_{s}=-W_{0}$ for $1<\xi<\xi_{E}$ (see Eq. (2.34)) yields, upon evaluation of the integrals,

$$
B=\left(W_{0} / \pi\right)\left(\xi_{E}^{2}-1\right)^{1 / 2} .
$$

It is easily checked that $W_{s}=0$ in $\xi>\xi_{E}$ since the contribution to the integral of the semicircular path segment above $\xi_{E}$ is $W_{0}$. Thus, Eq. (2.40) with $C \equiv 0$ and $A$ and $B$ defined by Eqs. (2.41) and (2.43), respectively, satisfies the boundary conditions (2.31)(2.34).

The problem is now in principle solved. To find an expression for $W$ in terms of $r$, $t$ and $\theta, \zeta$ has to be expressed in terms of $\gamma$ by means of Eq. (2.24), whereupon the result must be substituted in Eqs. (2.40) and (2.30). As is often the case with the SchwarzChristoffel transformation, unfortunately, it is not possible to invert Eq. (2.24) analytically. The information that has thus far been gathered is, however, sufficient to derive expressions for the stresses and the particle velocity in the vicinity of the moving crack tip. This is shown in the next section.

3. Stresses in the vicinity of the crack tip. Within the rectangular region in the $\theta-\beta$ plane shown in Fig. 2 the particle velocity $W$, as a function of $\beta$ and $\theta$, is governed by Laplace's equation (see Eq. (2.18)). The region contains a semi-infinite slit, whose tip is located at the point $D$. Let us consider the cylindrical coordinate system $z, p, \psi$, centered at $D$, and where $p$ represents the radial distance measured from $D$ while $\psi$ is the angle shown in Fig. 2. It is well known that the derivatives of a field quantity governed by Laplace's equation have a square root singularity at the tip of the slit. Thus, as the point $D$ is approached, $\partial W / \partial p$ and $(1 / p) \partial W / \partial \psi$, or $T_{p z}$ and $T_{\psi z}$, show square-root singularities. The forms of $T_{p z}$ and $T_{\psi_{z}}$ follow, for example, from the results presented by Sih [6] as

$$
\begin{aligned}
& T_{p z}=\left(K \cos \frac{1}{2} \psi\right) /(2 p)^{1 / 2}+\mathrm{O}\left(p^{1 / 2}\right), \\
& T_{\psi_{z}}=-\left(K \sin \frac{1}{2} \psi\right) /(2 p)^{1 / 2}+\mathrm{O}\left(p^{1 / 2}\right),
\end{aligned}
$$

where $K$ is the intensity factor.

To relate $K$ to $G(\gamma)$ we follow the steps outlined in the paper by Sih [6]. Thus, first Eqs. (3.1) and (3.2) are combined to form

$$
T_{p z}-i T_{\psi z} \sim\left[K /(2 p)^{1 / 2}\right] \exp (i \psi / 2)
$$

and then, since $\gamma-\gamma_{D}=p \exp (i \psi)$ (see Fig. 2), we find

$$
T_{p z}-i T_{\psi z} \sim\left[K / 2^{1 / 2}\left(\gamma-\gamma_{D}\right)^{1 / 2}\right] \exp (i \psi) .
$$

By comparing Eqs. (2.21) and (3.4) it follows that

$$
K\left(\gamma_{D}\right)=2^{1 / 2} \mu \lim _{\gamma \rightarrow \gamma D}\left(\gamma-\gamma_{D}\right)^{1 / 2} G^{\prime}(\gamma) .
$$


In the previous section the conformal mapping $\gamma=\omega(\zeta)$ was employed, (see Eqs. (2.23) and (2.24)) to map the rectangular region on the upper half of the $\zeta$-plane. In this mapping the point $D$ is mapped on the real axis, and its position is defined by $\xi_{D}$. Concomitant with the mapping, Eq. (3.5) becomes

$$
K\left(\xi_{D}\right)=2^{1 / 2} \lim _{\zeta \rightarrow \xi_{D}} \frac{\left[\omega(\zeta)-\omega\left(\xi_{D}\right)\right]^{1 / 2} F^{\prime}(\zeta)}{d \omega / d \zeta} .
$$

In general the function $d F / d \zeta$ is continuous at $\zeta=\xi_{D}$ and $d \omega / d \zeta$ vanishes as $\zeta \rightarrow \xi_{D}$. It is necessary to use L'Hospital's rule to obtain

$$
K\left(\xi_{D}\right)=\mu F^{\prime}\left(\xi_{D}\right) /\left[\omega^{\prime \prime}\left(\xi_{D}\right)\right]^{1 / 2} .
$$

By employing Eqs. (2.39) and (2.23), the intensity factor for the problem at hand is now easily evaluated as

$$
K=\frac{\mu\left(\xi_{P}+\kappa\right)\left(\xi_{Q}-\kappa\right)^{1 / 2}}{(1+\kappa)^{1 / 2}} \frac{\left(\xi_{P}+\xi_{Q}\right)^{1 / 2}}{\left(\left(1-\kappa^{2}\right)\left(1-\xi_{P}\right)^{2}\right]^{1 / 4}}\left[\frac{A}{\xi_{B}+\kappa}-\frac{B}{\xi_{E}-\kappa}\right] .
$$

It can be shown that $K$ is an even function of $\kappa$.

The remaining task is to employ Eqs. (3.1) and (3.2) to obtain expressions for the shear stress and the particle velocity in the vicinity of the crack tip. To this end we introduce a new set of polar coordinates $z, R, \varphi$ centered at the crack tip, as shown in Fig. 4 . The pertinent shear stress $\tau_{\varphi z}$ is related to $\tau_{r z}$ and $\tau_{\theta z}$ by

$$
\tau_{\varphi z}=-\tau_{r z} \sin \varphi-\tau_{\theta z} \cos \varphi .
$$

Denoting $\partial \tau_{r z} / \partial t$ by $T_{r z}$, we can derive the following expression for $T_{r z}$ by employing Eqs. (2.6a) and (2.19):

$$
T_{r z}=(\partial \beta / \partial r) \mu \operatorname{Re} G^{\prime}(\gamma) .
$$

Eliminating Re $G^{\prime}(\gamma)$ by means of Eq. (2.21), and employing Eqs. (3.1) and (3.2), we find, for small values of $p$,

$$
T_{r_{2}} \sim-\left(1-v^{2} / c_{T}{ }^{2}\right)^{-1 / 2}\left[K /(2 p)^{1 / 2} v t\right] \cos \frac{1}{2} \psi .
$$

Similarly,

$$
T_{\theta z} \sim\left[K /(2 p)^{1 / 2} v t\right] \sin \frac{1}{2} \psi .
$$

For small values of $R / v t$ the following relations can easily be derived:

$$
\begin{aligned}
& \theta \sim \kappa \pi+R \sin \varphi / v t, \\
& r \sim v t[1-(R / v t) \cos \varphi],
\end{aligned}
$$

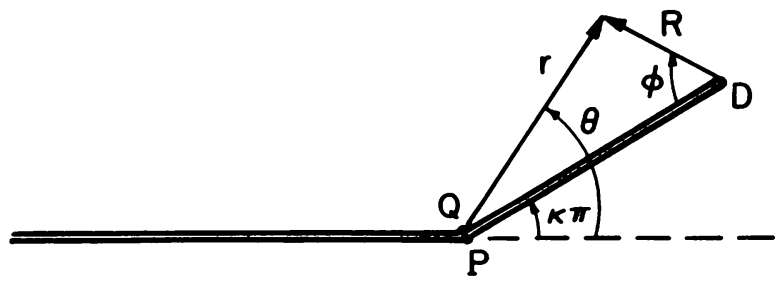

FIg. 4. Polar coordinates centered at the moving crack tip. 


$$
\beta \sim \beta_{D}+[(R / v t) \cos \varphi] /\left(1-v^{2} / c_{T}^{2}\right)^{1 / 2} .
$$

By writing $p$ and $\psi$ in terms of $\theta$ and $\beta$, and by employing Eqs. (3.13)-(3.15), expressions can be obtained relating $p, \sin (\psi / 2)$ and $\cos (\psi / 2)$ to $R, \varphi$ and $t$ respectively. Substitution of the results in Eqs. (3.11) and (3.12), integration with respect to $t$ and subsequent substitution of the results in Eq. (3.9) results in

$$
\tau_{\varphi z} \sim k_{\tau} \Phi_{\tau}\left(v / c_{T}, \varphi\right)(t / R)^{1 / 2}
$$

where

$$
\begin{aligned}
& k_{\tau}=\left(1-v^{2} / c_{T}^{2}\right)^{1 / 4} K / v^{1 / 2}, \\
\Phi_{\tau}\left(v / c_{T}, \varphi\right)= & \left\{\frac{\left[1-\left(v / c_{T}\right)^{2} \sin ^{2} \varphi\right]^{1 / 2}+\cos \varphi}{\left[1-\left(v / c_{T}\right)^{2}\right]\left[1-\left(v / c_{T}\right)^{2} \sin ^{2} \varphi\right]}\right\}^{1 / 2} \sin \varphi \\
& -\left\{\frac{\left[1-\left(v / c_{T}\right)^{2} \sin ^{2} \varphi\right]^{1 / 2}-\cos \varphi}{1-\left(v / c_{T}\right)^{2} \sin ^{2} \varphi}\right\}^{1 / 2} \cos \varphi .
\end{aligned}
$$

It should be noted that $\Phi_{\tau}$ does not depend on $\kappa$. The dependence of $\tau_{\varphi z}$ on $\varphi$ in the immediate vicinity of the crack tip thus is independent of the skew of crack propagation.

In Fig. 5 the function $\Phi_{r}$ is plotted versus $\varphi$ for various values of $v / c_{T}$. It is noted that the maximum of $\Phi_{r}$ moves out of the plane of crack propagation $(\varphi=\pi)$ as $v / c_{r}$ increases.

To compute the particle velocity in the vicinity of the crack tip we observe that

$$
\partial W / \partial t=-(r / t) \partial W / \partial r .
$$

Using (3.11) and integrating with respect to $t$, we obtain

$$
W \sim k_{W} \Phi_{W}\left(v / c_{T}, \varphi\right)(t / R)^{1 / 2}
$$

where

$$
k_{W}=-v^{1 / 2} K /\left(1-v^{2} / c_{T}^{2}\right)^{1 / 4} \mu
$$

and

$$
\Phi_{W}\left(v / c_{T}, \varphi\right)=\left\{\frac{\left[1-\left(v / c_{T}\right)^{2} \sin ^{2} \varphi\right]^{1 / 2}+\cos \varphi}{1-\left(v / c_{T}\right)^{2} \sin ^{2} \varphi}\right\}^{1 / 2} .
$$

4. Propagation of the crack in its own plane. For this case, which corresponds to $\kappa=0$, it follows from Eq. (2.27) that $\xi_{P}=\xi_{Q}$. The conformal mapping (2.28) now reduces to

$$
\gamma=i \pi-2 i \arctan \left[\left(\zeta^{2}-1\right)^{1 / 2} /\left(1-\xi_{P}{ }^{2}\right)^{1 / 2}\right] .
$$

This relation can be inverted to yield

$$
\zeta^{2}-1=-\left(1-\xi_{P}{ }^{2}\right) \operatorname{coth}^{2}[(\beta+i \theta) / 2] .
$$

The point $D$ now maps into the origin of the $\zeta$-plane. By the use of Eq. (4.2) the mapping of $D$ yields an equation for $\xi_{P}$ and $\xi_{Q}$ which can be solved as

$$
\xi_{P}=\xi_{Q}=2^{1 / 2}\left(1+c_{T} / v\right)^{-1 / 2}
$$

By means of Eq. (4.2) we also find 


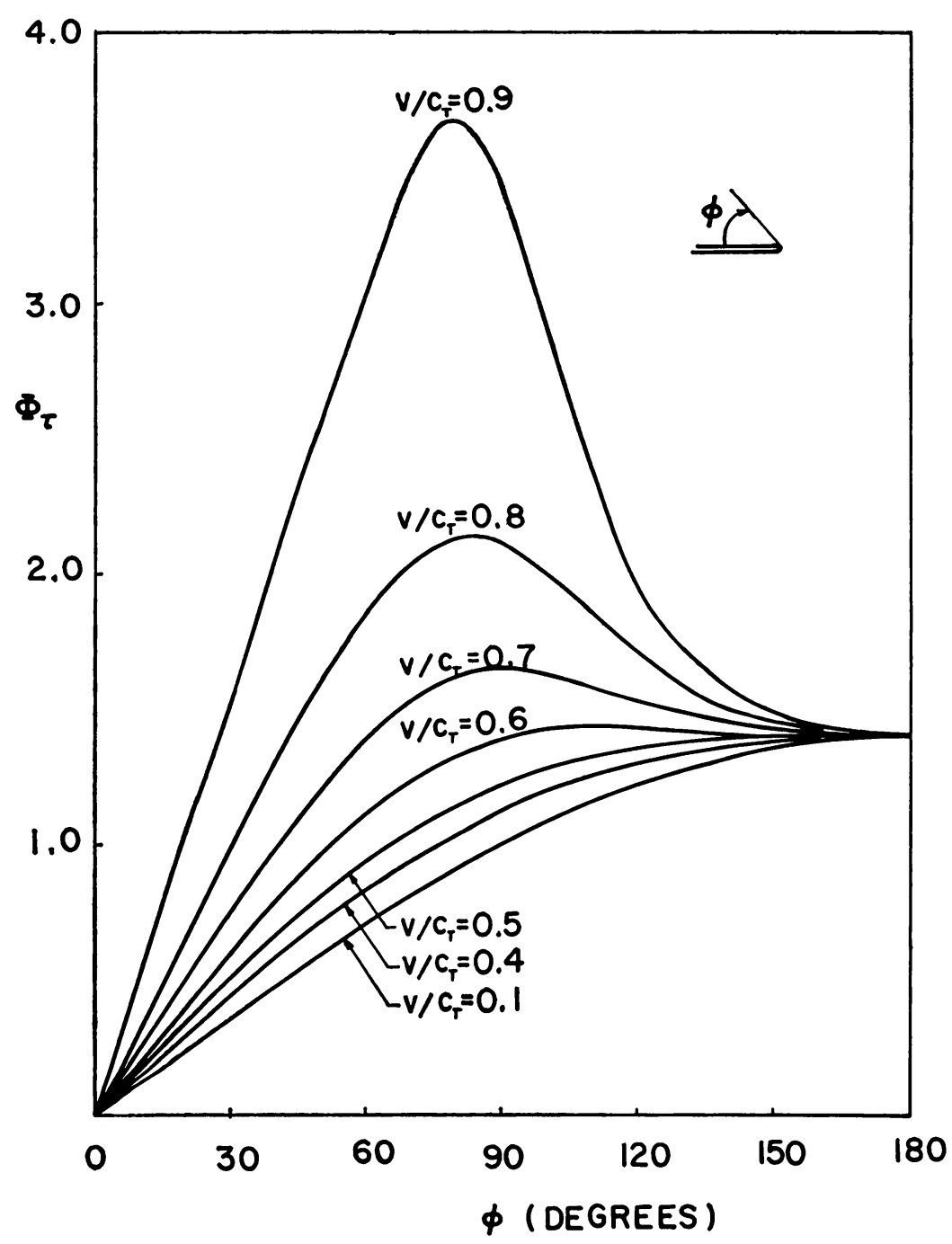

FIG. 5. Dependence of $\tau_{\varphi z}$ in the vicinity of the crack tip on the polar angle $\varphi$.

$$
\xi_{B}=\xi_{E}=\frac{2^{1 / 2}(1-\sin \alpha)^{1 / 2}\left(c_{T} / v+\sin \alpha\right)^{1 / 2}}{\left(c_{T} / v+1\right)^{1 / 2} \cos \alpha} .
$$

The constants $A$ and $B$ easily follow as

$$
A=-B=\frac{W_{0}}{\pi}\left(\frac{c_{T} / v-1}{c_{T} / v+1}\right)^{1 / 2} \frac{1-\sin \alpha}{\cos \alpha} .
$$

Substitution of Eqs. (4.3)-(4.5) into Eq. (3.8) yields

$$
\left.K\right|_{\alpha=0}=\frac{2^{1 / 2} \mu W_{0}}{\pi c_{T}} \frac{\left(c_{T}-v\right)^{1 / 2}(1-\sin \alpha)^{1 / 2} c_{T}{ }^{1 / 2} v^{1 / 2}}{\left(c_{T}{ }^{2}-v^{2}\right)^{1 / 4}\left(1+v \sin \alpha / c_{T}\right)^{1 / 2}}
$$

The corresponding expression for $\tau_{\varphi z}$ can be shown to agree with an expression derived earlier in [9] in the vicinity of the crack tip for a crack propagating in its own plane.

For a stationary crack $(v \equiv 0)$, the factor $k_{r}$ and the function $\Phi(\varphi)$ follow immediately 
from Eqs. (3.17), (4.6) and (3.18). The corresponding $\tau_{\varphi z}$ can be shown to agree with the results of $[10]$.

5. Singularity at the corner. The stress $\tau_{\theta_{z}}$ in the vicinity of the corners at the points $P$ and $Q$ can also be obtained by a limiting process in the $\zeta$-plane. Since the manipulations are straightforward and similar to the ones carried out in [4], they will not be recorded here. In the vicinity of $P$ we find

$$
\tau_{\theta_{z}} \sim \frac{1}{2} \frac{\mu}{\kappa c}\left(1-\xi_{P}^{2}\right)^{-1 / 2}\left[\frac{A}{\xi_{B}-\xi_{P}}-\frac{B}{\xi_{P}+\xi_{E}}\right]\left(\frac{2 c t}{r}\right)^{\kappa /(1+\kappa)} \sin \left(\frac{\pi+\theta}{1+\kappa}\right) .
$$

The general form of the dependence on $r$ agrees with what is usually found at the vertex of a wedge. It should be noted that the stress is not singular if $\kappa \leq 0$. A similar expression can be derived for the stress in the vicinity of the point $Q$.

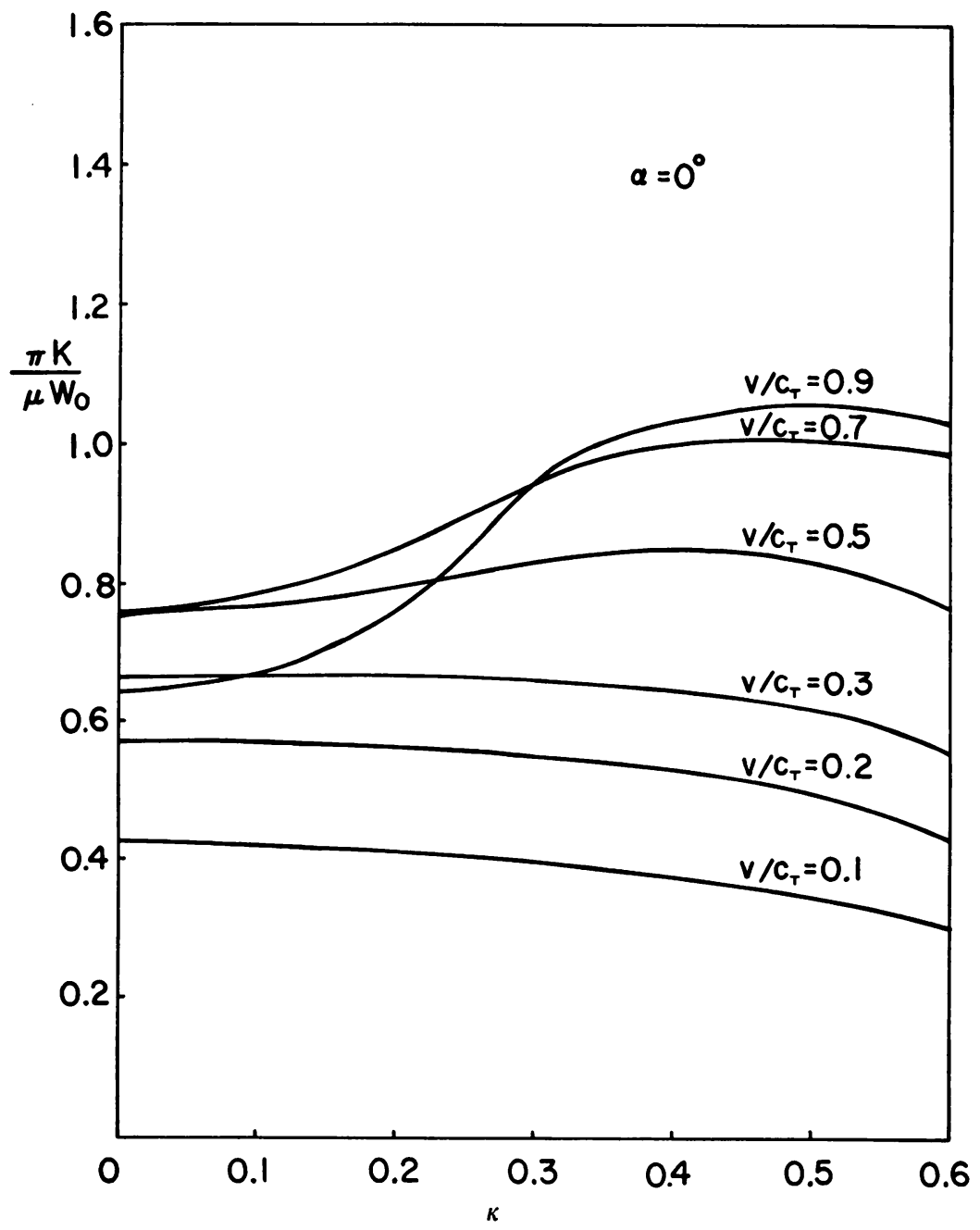

Fig. 6. The factor $K$ as a function of the angle of skew; $\alpha=0^{\circ}$. 


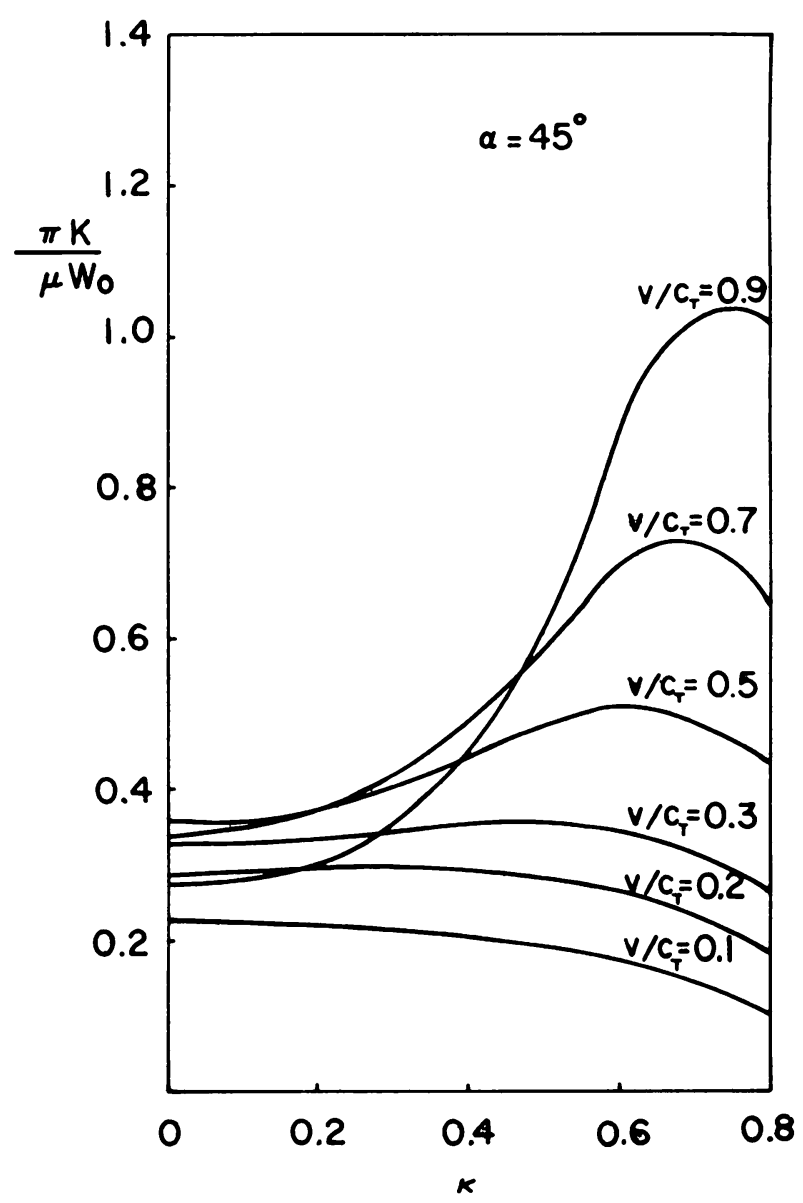

Fig. 7. The factor $K$ as a function of the angle of skew; $\alpha=45^{\circ}$.

6. Results. The intensity factor $K$, which appears in the expressions for $\tau_{\varphi s}$ and $W((3.16)$ and (3.20), respectively), has been plotted in Figs. 5 and 6 versus the parameter $\kappa$ which defines the angle of skew. The diagrams show that for small values of $v / c_{T}$ the maximum of $K$ occurs for $\kappa=0$, i.e. for propagation of the crack in its own plane. As the speed of crack propagation increases beyond approximately $v / c_{T}=0.3$ for an angle of incidence $\alpha=0^{\circ}$, and a value somewhat smaller than $v / c_{T}=0.2$ for $\alpha=45^{\circ}$, the maxima of $K$ occur at increasing values of $\kappa$. When $v / c_{T}$ approaches unity the maxima move to $\kappa=0.5$ and $\kappa=0.75$ for $\alpha=0$ and $\alpha=45^{\circ}$, respectively, i.e. toward the point corresponding to $E$ in Fig. 1 .

\section{REFERENCES}

[1] J. D. Achenbach, Extension of a crack by a shear wave, Z. angew. Math. und Phys. 21, 887-900 (1970)

[2] J. D. Achenbach and R. J. Nuismer, Fracture generated by a dilatational wave. Int. J. Fract. Mech. 7, 77-88 (1970)

[3] J. W. Miles, Homogeneous solutions in elastic wave propagation, Quart. Appl. Math. 18, 37-59 (1959)

[4] J. D. Achenbach, Shear waves in an elastic wedge, Int. J. Solids and Structures 6, 379-388 (1970) 
[5] N. I. Muskhelishvili, Some basic problems of the mathematical theory of elasticity (trans. J. R. M. Radok), Noordhoff, Groningen, 1963

[6] G. C. Sih, Stress distribution near internal crack tips for longitudinal shear problems, J. Appl. Mech. 32, 51-58 (1965)

[7] G. F. Carrier, M. Krook and C. E. Pearson, Functions of a complex variable, McGraw-Hill, New York, 1966

[8] G. C. Sih, Boundary problems for longitudinal shear cracks, in Developments in theoretical and applied mechanics (W. A. Shaw, ed.), Vol. 2, 117-130 (1965)

[9] J. D. Achenbach, Crack propagation generated by an incident shear wave, Tech. Rep. 69-4, Dept. of Civil Engineering, Northwestern University, 1969.

[10] G. H. Handelman and L. A. Rubenfeld, Diffraction of horizontal shear waves by a half plane, J. Appl. Mech. 36, 873-875 (1969) 